\title{
Optimal coding for naturally occurring whisker deflections
}

\author{
Verena Vanessa Hafner ${ }^{1}$, Miriam Fend ${ }^{1}$, Max Lungarella ${ }^{1}$, \\ Rolf Pfeifer ${ }^{1}$, Peter König ${ }^{2}$, and Konrad Paul Körding ${ }^{2} \star \star$ \\ ${ }^{1}$ Artificial Intelligence Laboratory, Dept. of Inf. Tech., University of Zurich \\ ${ }^{2}$ Institute of Neuroinformatics, University of Zurich / ETH Zurich \\ Winterthurerstr. 190, 8057 Zurich, Switzerland \\ \{vhafner,fend, lunga, pfeifer\}@ifi.unizh.ch \\ \{peterk, koerding\}@ini.phys . ethz.ch
}

\begin{abstract}
It is largely unknown how the properties of the somatosensory system relate to the properties of naturally occurring whisker deflections. Here, we analyse representations of simulated neurons that have optimally sparse activity in response to recorded reflections of a rat whisker from surfaces of everyday objects. These representations predict a number of interesting properties of neurons in the somatosensory system that have not been measured yet.
\end{abstract}

\section{Introduction}

For about a century it has been known that the vibrissae or whiskers provide an important source of information to rats and other rodents [1]. In particular, rats can distinguish surface properties purely on the basis of cues from their whiskers [2][3]. Rats can furthermore use their whiskers to discriminate objects [4]. As the rat explores its environment, its whiskers are moved over various shapes and surfaces. The whisker deflections caused by these stimulations define the input to the rat's somatosensory system. Although a large number of studies analyses the electrophysiology in this system [5][6][7], the relevant features of its input have remained unknown.

It is evidently difficult to analyse complex natural stimuli. Fortunately, many studies have addressed the properties of natural stimuli in the visual [8][9] and the auditory domain [10][11]. Simulated neurons with optimally sparse activity reproduce much of the properties of neurons in the early visual and auditory areas. Optimally sparse [12] in this context means that the neurons often have an activity close to zero and then sometimes have very high activity. Drawing upon this inspiration, we analyse the somatosensory system with similar methods.

In this paper, we examine the statistics of natural stimuli to the somatosensory system. To do so, we built an artificial whisker system, with a real rat whisker attached to a capacitor microphone. This is in contrast to previous

\footnotetext{
* Current working address: Neuroscience Research Institute (AIST), Tsukuba, Japan ** Current working address: Institute of Neurology, UCL, London, UK
} 
robotics studies, that used simple whisking devices measuring distances or contact only [13][14][15], but do not capture the rich information picked up by natural whiskers.

We analyse if the neurons in the vibrissal system can also be understood in terms of leading to sparse activity in response to these natural inputs. The data coming from our artificial whisker system is analysed in the spectro-temporal domain. Simulated neurons optimally coding for these data are analysed and generate predictions about neurons in the somatosensory system.

\section{The Artificial Whisker System}

\subsection{Hardware}

The desired artificial whisker should be functionally comparable to a natural rat whisker and therefore be sensitive to small amplitude deflections and fast oscillations. We attached a rat whisker to the diaphragm of a capacitor microphone using cyanoacrylic super-glue. The change in voltage resulting from whisker deflections is preamplified and digitally recorded. This technique allows us to measure fast oscillations of the whisker even if the amplitude is very low. A schematic drawing of the device is shown in figure 1 left.
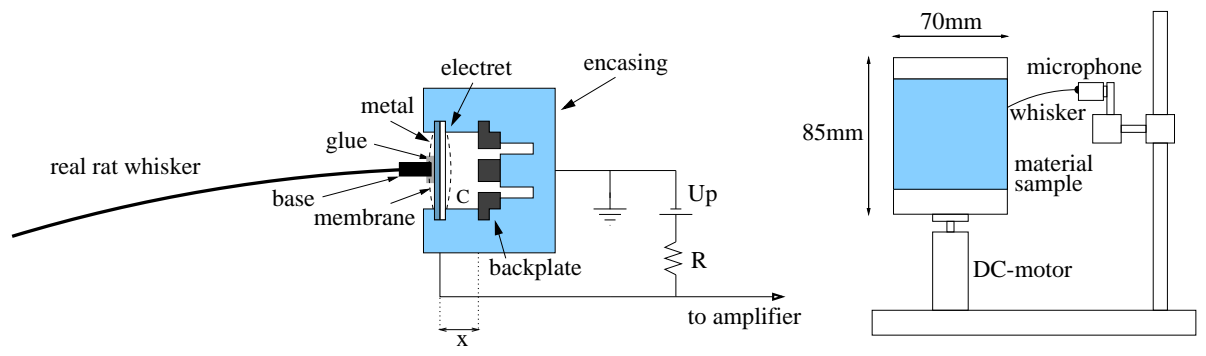

Fig. 1. Left: Basic schematic of the artificial whisker with a capacitor microphone being its main component. The deflection of the membrane is measured by the change of capacitance. The related change of voltage is fed into a preamplifier circuit. Right: Experimental device used to perform some of the experiments described and analysed in this paper.

\subsection{Data Obtained}

We consider two distinct datasets. Sandpaper data set: we recorded the deflections of whiskers that touched a cylinder rotating with constant speed covered with sandpaper (see figure 1 right). We used a set of natural rat whiskers of different length $(37 \mathrm{~mm}-51 \mathrm{~mm})$ and distance $(20 \mathrm{~mm}-45 \mathrm{~mm})$ to the cylinder. Natural 
object data set: we recorded deflections from a single whisker being manually swept over nine objects and surfaces (fur, leaves, etc.). Data are sampled at $4096 \mathrm{~Hz}$. Typical recordings from the two data sets can be seen in figure 2 .
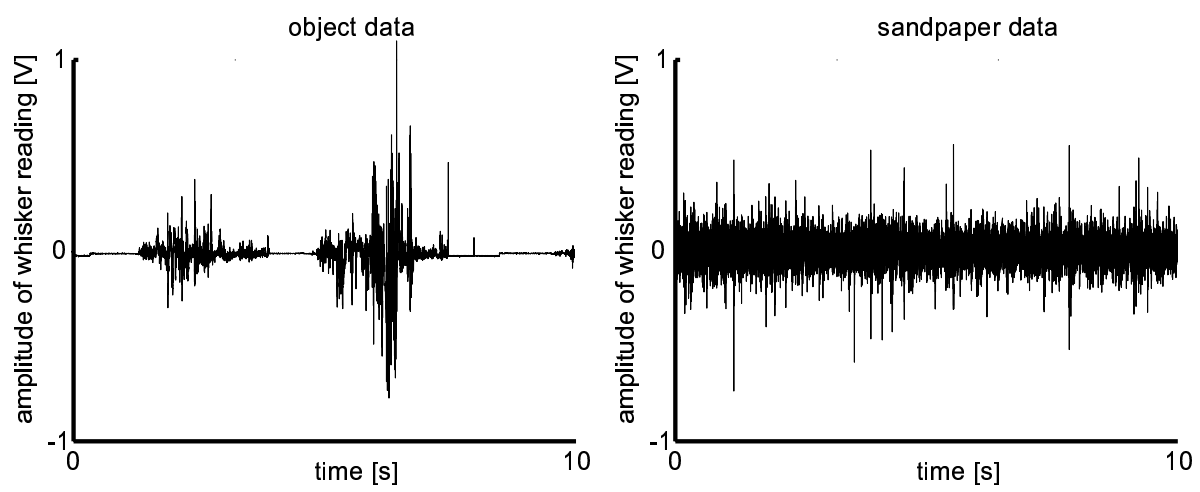

Fig. 2. Data received directly from the artificial whisker system while moving the whisker over an object (natural object data set, left) or while rotating a cylinder covered with sandpaper along the whisker (sandpaper data set, right).

\section{Processing Methods}

\subsection{Representation of the Data}

Time varying data are conveniently analysed in spectrogram space, the space spanned by frequency and time. This representation is particularly useful for the whisker system since rats are able to discriminate surfaces of different spatial frequencies [3]. We thus transform the input signals into spectrograms using methods adapted to the analysis of temporally changing signals which are also used for auditory processing. They are available as a matlab package ("NSL Tools" [16]). The resolution on the tonotopic axis is 64 points, covering a frequency range from $4.7 \mathrm{~Hz}$ to $185.5 \mathrm{~Hz}$. In figure 3 , three typical samples of such transformed whisker data (recorded with the natural object data set) can be seen. These spectrograms show that whisker deflections lead to a largely conserved frequency-time response. We cut the spectrogram data in windows of $250 \mathrm{~ms}$ each, overlapping by $10 \mathrm{~ms}$. The temporal resolution of these windows is 25 points.

\subsection{Spectrotemporal Receptive Fields}

For the learning studies, the spectrograms are first compressed by a principal component analysis $(\mathrm{PCA})$ using the first $n_{P C A}=100$ principal components 


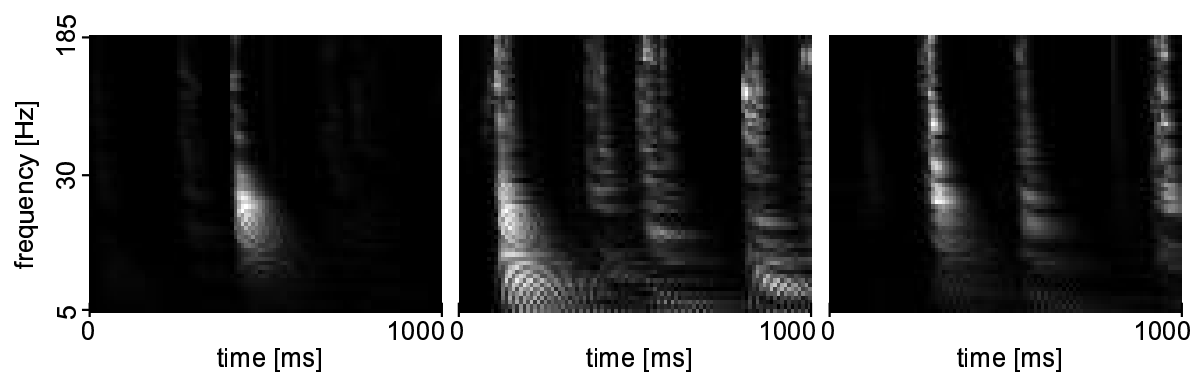

Fig. 3. Sample spectrogram of whisker data recorded with the natural object data set. The frequency axis logarithmically ranges from $4.7 \mathrm{~Hz}$ to $185.5 \mathrm{~Hz}$ while time runs from 0 to $1000 \mathrm{~ms}$.

(out of $25 \times 64=1600$ ). These components capture more than 96 per cent of the variance. We subsequently assemble a set of 2025 samples of natural object data spectrograms, and a second set of 1050 samples of sandpaper data spectrograms.

A set of 32 simulated neurons is trained to optimally code for each of these data.

The activity of the neurons is defined as

$$
A_{i}(t)=I(t) W_{i}(t)
$$

where $A_{i}$ is the activity, $W_{i}$ is the weight vector of the neuron $i . I(t)$ is the input vector of length $n_{P C A}$ shared by all neurons. The weights connecting each neuron to the spectrogram data, are optimised by scaled gradient descent to minimise the following loss function:

$$
\Psi_{\text {total }}=\Psi_{\text {cauchy }}+\Psi_{\text {std }}+\Psi_{\text {decorr }}, \text { with: }
$$

- Cauchy: $\Psi_{\text {cauchy }}=\frac{1}{n} \sum_{i}<\ln \left(1+A_{i}(t)^{2}\right)>_{t}$,

with $\langle\cdot\rangle_{t}$ being the average over time $t$

- Standard deviation: $\Psi_{s t d}=\frac{1}{n} \sum_{i}\left(\sigma_{A_{i}}-1\right)^{2}$

- Decorrelation: $\Psi_{\text {decorr }}=\frac{4 \sum_{i, j} C_{i j}^{2}}{(n-2)(n-1)}$,

with $C=\operatorname{cov}(A)$ being the $n \times n$ covariance matrix of $A$

While the $\Psi_{\text {cauchy }}$ measures the sparseness of the responses, the two other loss functions ensure the standard criterion used in Independent Component Analysis (ICA) and sparse coding studies that the output variances should be unitary and the output covariances should be vanishing. 


\section{Results}

Simulated neurons are optimised to sparsely encode naturally occurring whisker deflections. Figure 4 shows the general properties of the resulting spectrotemporal receptive fields. Most of the analysed neurons are localised in time, and some are also localised in frequency (figure 4, plots A, F, H, I, and J).

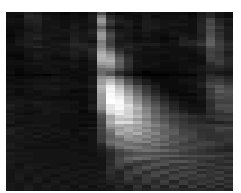

A

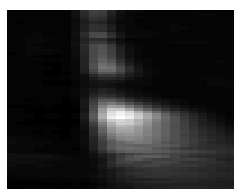

$\mathbf{F}$

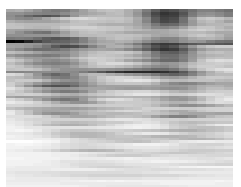

B

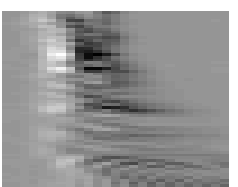

G

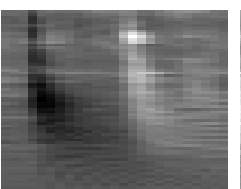

C

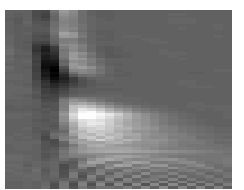

$\mathbf{H}$

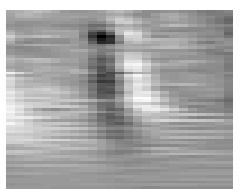

D

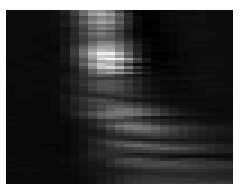

I

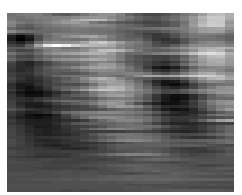

$\mathbf{E}$

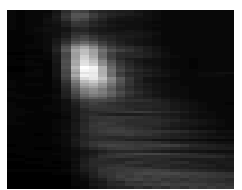

$\mathbf{J}$

Fig. 4. Five samples each (top row: sandpaper data set, bottom row: objects data set) of typical colour-coded spectrotemporal receptive fields out of 32 neurons. $y$-axis: frequency $(4.7 \mathrm{~Hz}$ to $185.5 \mathrm{~Hz}), x$-axis: time (0 to $250 \mathrm{~ms})$.

To further quantify this property, we introduce two measures of localisedness (figure 5). For the analysis, we calculate the average energy over time, and the frequency for each receptive field. We also measure the width of the maximum peak at half the peak value for time localisation, and the octaves $\log \left(f_{l} / f_{h}\right)$ for frequency localisation. More than 87 per cent of the receptive fields from the object data set have a localisation measure in time of less than $100 \mathrm{~ms}$. The receptive fields from the sandpaper data set have a localisation measure in time of less than $100 \mathrm{~ms}$ in only 68 per cent. The percentage of neurons with a tuning width of less than 3 octaves, and thus selective to frequency, is 43 per cent in the object data set and 46 per cent in the sandpaper data set

This is in analogy to sparse simulated neurons in the visual system that obtain localised receptive fields in space and orientation [8]. In addition to this, they are often tuned to changes or even modulations of the energy of the input over time.

This property might be useful for tactile texture recognition. There is some influence of the choice of the stimulus set. The sandpaper data show a stronger degree of modulation selectivity while the natural textures data show a stronger specificity to frequency. To which degree these properties depend on specific properties of the datasets remains an issue for further research. 

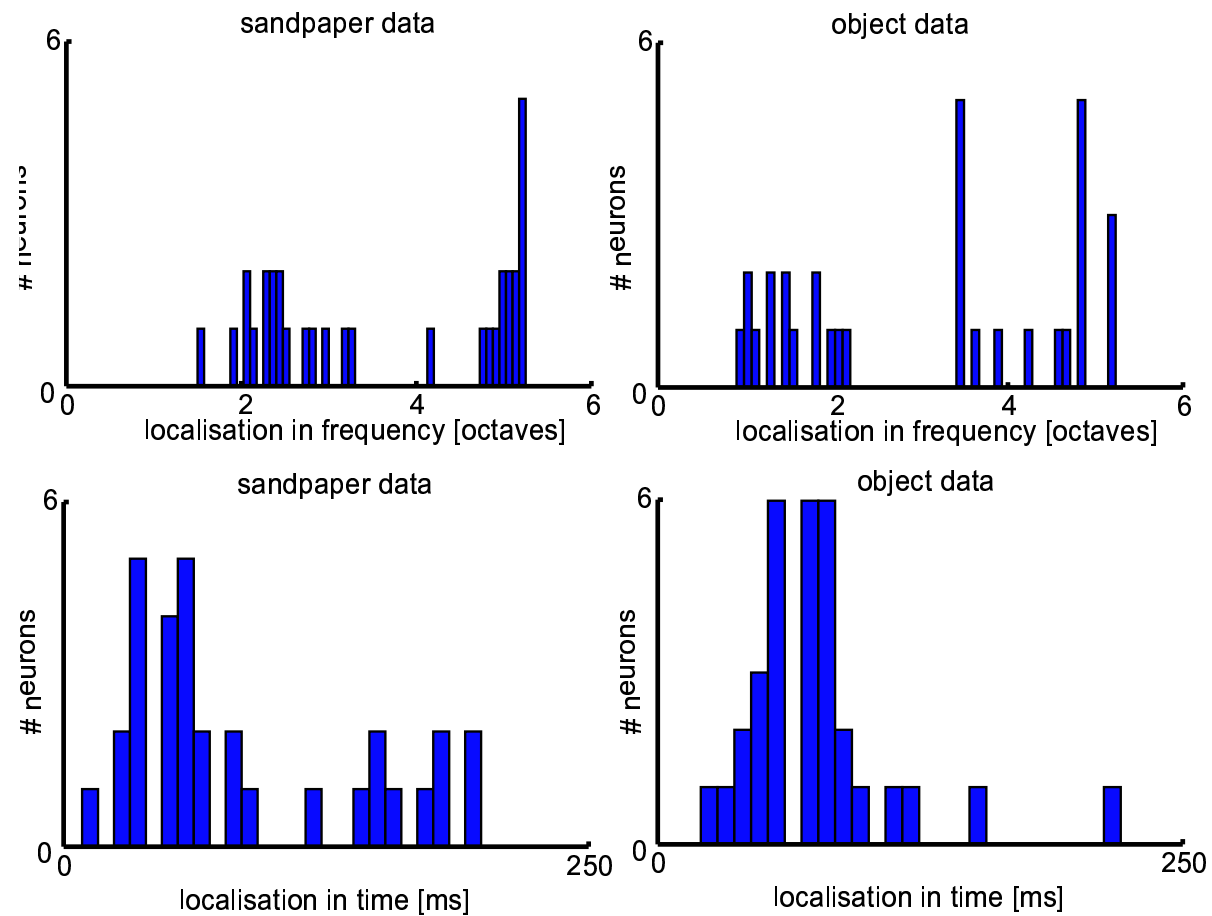

Fig. 5. Histograms showing the localisedness of the spatiotemporal receptive fields for frequency in octaves (top row) and for time in ms (bottom row) for both data sets.

\section{Discussion}

Our simulations investigate the optimal coding of naturally occurring whisker deflections. We proceed with the discussion of the properties of the whisker representation in the rodent brain, and based on our results we make predictions of the properties of neurons that have not been measured yet in physiological experiments.

\subsection{Choice of the Representation}

It is not clear which stimulus features allow rats to perform discrimination tasks involving high spatial frequencies and neither are there physiological studies that analyse the time frequency properties of neurons in the somatosensory system. Since the waveforms of high frequency signals are not a good indicator of their properties (in speech for example, we can scramble the phase spectrum without changing the perceived sounds) a different representation is necessary. Auditory and somatosensory systems share similar temporal stimulus properties (data not shown). Drawing upon this inspiration, we thus represent the whisker data as 
spectrograms the same way auditory data are typically represented. By training the simulated neurons to have sparse activities on these inputs, we extract salient features from the obtained natural stimuli.

\subsection{Physiological Studies}

Biological studies have focused mainly on low-frequency stimulation of one or more whiskers and have studied neuronal parameters such as latency of thalamic response [17], cortical response [18][19], and ON or OFF response magnitude [20][21]. The stimulations used for most biological studies consist of air puffs or ramp-hold deflections, either in single trials or with frequencies around the natural whisking frequency of $8 \mathrm{~Hz}$. There are no published studies that investigate in the response pattern of neurons in the somatosensory system to stimuli of frequencies between 20 and $200 \mathrm{~Hz}$. There is however some preliminary evidence that neurons show complex behaviour in this range (R.S. Petersen, M.E. Diamond, personal communications). The lack of experimental data is particularly surprising since it is known that rats can discriminate surfaces and textures with high spatial frequency, translating into high frequency of whisker stimulations $[3]$.

\subsection{Predictions}

Our study investigates how such frequencies of obvious behavioural relevance can optimally be encoded by neural representations. Simulated neurons optimally coding for natural stimuli can be viewed as predictions of the, yet unmeasured, neural properties at higher stimulation frequencies.

This study predicts that some neurons in the somatosensory system should not code for the frequency of the whiskers stimulation but rather code for modulation frequencies. Such cells might actually be better detectors for surface texture properties than cells that are just localised in spectrum. Most of them should have temporally localised responses and show some selectivity to stimula-

tion frequency. These predictions can be tested in experimental studies probing the somatosensory system with spectrotemporal patterns.

Acknowledgements This research has been supported by the IST-2000-28127 European project (AMOUSE), by grant \#20-068198.02 of the Swiss National Science Foundation (VVH), and by the Collegium Helveticum for the dialogue of the sciences (KPK). The natural rat whiskers were kindly provided by Mathew Diamond at SISSA, Cognitive Neuroscience sector, Trieste, Italy.

\section{References}

1. S. B. Vincent. The function of the vibrissae in the behavior of the white rat. Behavior Monographs, 1(5):1-81, 1912. 
2. E. Guic-Robles, C. Valdivesco, and G. Guajardo. Rats can learn a roughness discrimination using only their vibrissal system. Behavioral Brain Research, 31:285289, 1989.

3. G. E. Carvell and J. Simons. Biometric analyses of vibrissal tactile discrimination in the rat. Journal of Neuroscience, 10(8):2638-2648, 1990.

4. M. Brecht, B. Preilowski, and M. M. Merzenich. Functional architecture of the mystacial vibrissae. Behavioral Brain Ressearch, 84(1-2):81-97, 1997.

5. E. Ahissar and A. Arieli. Figuring space by time. Neuron, 32:185-201, October 2001.

6. K. D. Miller, D. J. Pinto, and D. J. Simons. Processing in layer 4 of the neocortical circuit: new insights from visual and somatosensory cortex. Current opinion in neurobiology, 11:488-497, 2001.

7. C. I. Moore, S. B. Nelson, and M. Sur. Dynamics of neuronal processing in rat somatosensory cortex. Trends in Neurosciences, 22(11):513-520, 1999.

8. B. A. Olshausen and D. J. Field. Emergence of simple-cell receptive field properties by learning a sparse code for natural images. Nature, 381(6583):607-609, 1996.

9. J. H. van Hateren and D. L. Ruderman. Independent component analysis of natural image sequences yields spatio-temporal filters similar to simple cells in primary visual cortex. Procedings of the Royal Society London B, 265:2315-2320, 1998.

10. M. S. Lewicki. Efficient coding of natural sounds. Nature Neuroscience, 5(4):35663, 2002. 1097-6256 Journal Article.

11. K. P. Körding, P. König, and D. J. Klein. Learning of sparse auditory receptive fields. In Proceedings of the International Joint Conference on Neural Networks (IJCNN), 2002.

12. H. B. Barlow. Single units and sensation: a neuron doctrine for perceptual psychology? Perception, 1:371-394, 1972.

13. D. Jung and A. Zelinsky. Whisker-based mobile robot navigation. Proceedings of the IEEE/RSJ International Conference on Intelligent Robots and Systems (IROS), 2:497-504, 1996.

14. R. A. Russell. Using tactile whiskers to measure surface contours. In Proceedings IEEE International Conference on Robotics and Automation, pages 1295-1300, 1992.

15. M. Kaneko, K. Kanayama, and T. Tsuji. Active antenna for contact sensing. IEEE Transactions on Robotics and Automation, 14(2):278-291, 1998.

16. NSL Tools. http://www.isr.umd.edu/CAAR/pubs.html, Neural Systems Laboratory, University of Maryland, College Park, 1998.

17. R. Sosnik, S. Haidarliu, and E. Ahissar. Temporal frequency of whisker movement. I. Representations in brain stem and thalamus. Journal of Neurophysiology, 86:339353, 2001.

18. E. Ahissar, R. Sosnik, K. Bagdasarian, and S. Haidarliu. Temporal frequency of whisker movement. II. Laminar organization of cortical representation. Journal of Neurophysiology, 86:354-367, 2001.

19. E. Ahissar, R. Sosnik, and S. Haidarliu. Transformation from temporal to rate coding in a somatosensory thalamocortical pathway. Nature, 406:302-305, 2000.

20. H. T. Kyriazi, G. E. Carvell, and D. J. Simons. Off response transformations in the whisker/barrel system. Journal of Neurophysiology, 72(1):392-401, 1994.

21. J. A. Hartings, S. Temereanca, and D. J. Simons. High responsiveness and direction sensitivity of neurons in the rat thalamic reticular nucleus to vibrissa deflections. Journal of Neurophysiology, 83:2791-2801, 2000. 